\title{
The Stellar Population of the Thin Disk
}

\author{
Carlos Allende Prieto ${ }^{1} \dagger$ \\ ${ }^{1}$ Mullard Space Science Laboratory, University College London, \\ Holmbury St. Mary, RH5 6NT , Surrey, United Kingdom \\ email: callende@astro.as.utexas.edu
}

\begin{abstract}
We discuss recent observations of stars located close to the symmetry plane of the Milky Way, and examine them in the context of theories of Galaxy formation and evolution. The kinematics, ages, and compositions of thin disk stars in the solar neighborhood display complex patterns, and interesting correlations. The Galactic disk does not seem to pose any unsurmountable obstacles to hierarchical galaxy formation theories, but a model of the Milky Way able to reproduce the complexity found in the data will likely require a meticulous study of a significant fraction of the stars in the Galaxy. Making such an observational effort seems necessary in order to make a physics laboratory out of our own galaxy, and ultimately ensure that the most relevant processes are properly understood.
\end{abstract}

Keywords. Keyword1, keyword2, keyword3, etc.

\section{Introduction}

Large galaxies naturally produce disks. Radiative cooling of the gas and angular momentum conservation lead the early evolution of galaxies through dissipative collapse and disk formation. Disks are frequently observed in galaxies, even at high redshift (Förster Schreiber et al. 2006), and the Milky Way does not seem unique at all in showing a dual disk, with a distinct thin and thick components (Dalcanton \& Bernstein 2002).

Early attempts to place the Galactic thin disk in the context of a $\Lambda$-CDM universe exposed a number of problems. The number of observed surviving satellites appeared far too small compared to simulations. This problem is somewhat alleviated after the Sloan Digital Sky Survey (SDSS) has identified many new low-surface brightness galaxies in the immediate Galactic neighborhood (but see Koposov et al. 2008). It was also deemed hard for the disk to survive for as long as the observations suggested, 8-10 Gyr, and in particular to stay thin (Tóth \& Ostriker 1992, Kauffmann \& White 1993). More recent appraisals, however, indicate that as many as $85 \%$ of disk galaxies have not been involved in a merger with a mass ratio larger than 0.5 since redshift $\sim 1-1.5$, or approximately in the last 8 Gyr (Koda et al. 2009). A higher gas fraction in the accreted building blocks seems to favor disk survival (Hopkins et al. 2009).

The structure of galaxy disks is usually studied measuring surface brightness distributions. Anomalies such as spiral arms and HII regions are smoothed out, taking deprojected azimuthal averages in nearly face-on galaxies, modeling the radial dependence of the light distribution with exponential profiles (see, e.g., Aguerri et al. 2000; Prieto et al. 2001). Edge-on galaxies are, in turn, used to study the light distribution perpendicular to the plane. In the Milky Way, the spatial distribution of stars is studied using deep imaging surveys - counting stars and exploiting photometric calibrations to estimate the luminosity of the main-sequence as a function of color (e.g. Juric et al. 2008).

$\dagger$ Present address: Instituto de Astrofísica de Canarias, Vía Láctea S/N, La Laguna 38205, Tenerife, Spain. 
Being insiders to the Galaxy provides some advantages; for example, we can measure in detail the properties of individual stars using spectroscopy. Modern surveys employ cameras with a very broad dynamical range, and massive multiplexing capabilities for spectroscopy (see, e.g., Gunn et al. 1998, 2005; Onaka et al. 2008), making it feasible to obtain large data sets fast.

\section{Main structural components of the Milky Way}

There are multitude of studies of the main Galactic components using star counts. Two recent studies by Cabrera-Lavers et al. (2007) and Jurić et al. (2008) based on 2MASS (Skrutskie et al. 2006) and the SDSS (Abazajian et al. 2009), respectively, sample quite well the Galactic thin disk. These surveys are dominated by late-type (mainly $\mathrm{K}$ and $\mathrm{M}$ dwarf) stars, and their scale height is found to be about $200-300$ pc. There is no consensus on the (radial) scale length of the thin disk, and estimates range between 2.5 to $3.5 \mathrm{kpc}$, but the larger distances involved make this measurement harder. The thin disk is thought to contribute about $85 \%$ of the stars in the Galactic plane.

It is important to emphasize that the disk scale heights are expected to vary depending on where we look (Bilir et al. 2008), as the potential is far from perfectly smooth and axisymmetric. More dramatic is the variation of the scale height of the thin disk with age. Thus, Maíz-Apellaniz (2001) finds $h \sim 35$ pc from OB type stars - a value nearly 10 times smaller than determinations from late-type stars. This strong age dependence is also imprinted in the distribution of M-dwarfs observed spectroscopically in the SDSS, and West et al. $(2004,2006,2008)$ find a decreasing fraction of active stars (the youngest) as they sample farther away from the Galactic plane. Further evidence is also seen in the stellar kinematics in the solar vicinity, which are discussed in the next section.

\section{Stellar kinematics in the Solar Neighborhood}

The combination of Hipparcos (and Tycho) astrometry with radial velocities from highresolution spectroscopy has provided detailed coordinates in phase space for stars in the solar neighborhood ( $<100$ pc from us). Observations with the CORAVEL spectrographs (Baranne et al. 1979) have been used to study large samples of giants (Famaey et al. 2005) and especially F- and G-type dwarfs (the Geneva-Copenhagen survey, described in Nordström et al. 2004 and Holmberg et al. 2007, 2009).

The velocity distributions of nearby stars show plenty of structure. We discuss structure in more detail in the following section, and here we focus on other characteristics. Each of the velocity components of the thin disk ( $U, V$ and $W$ for the radial, azimuthal, and vertical components in a cylindrical coordinate system; see Fig. 1) shows distributions that increase in width with age. The scatter in the vertical velocities $(W)$ shows the sharpest and smoothest rise with age of the three velocity components from $\sigma \sim 8 \mathrm{~km}$ $\mathrm{s}^{-1}$ for stars that are $\sim 1$ Gyr old to roughly $30 \mathrm{~km} \mathrm{~s}^{-1}$ for stars $\sim 10$ Gyr old. This is another way of looking at the increase in scale height with time.

For a Mestel disk (surface density $\Sigma \propto R^{-1}$, where $R$ is the galactocentric distance), assuming an isothermal sheet $\rho \propto \cosh ^{-2}(z)$, embedded on a spherical halo $\left(\rho \propto r^{-2}\right.$, where $r$ is the radial coordinate), the disk rotational velocity $V_{\text {rot }}$ is constant, and the vertical velocity dispersion can be written

$$
\sigma^{2}=\frac{1}{2} V_{\text {rot }}^{2} \frac{h}{R_{\odot}}\left(\mu+\lambda(1-\mu) \frac{h}{R_{\odot}}\right),
$$

(Tóth \& Ostriker 1992), where $\lambda \equiv \int x^{2} \cosh ^{-2}(x) d x \simeq 1.645, \mu(R)$ is the enclosed mass 
ratio (disk/total, about 0.35 for the Milky Way), and adopting $V_{\text {rot }} \simeq 220 \mathrm{~km} \mathrm{~s}^{-1}$,

$$
\sigma^{2} \simeq 24200 \frac{h}{R_{\odot}}\left(0.35+1.10 \frac{h}{R_{\odot}}\right) .
$$

This indicates that a range in $\sigma$ between 8 and $30 \mathrm{~km} \mathrm{~s}^{-1}$, as observed for stars between 1 and 10 Gyr should be matched by an excursion in the scale height between 60 and $600 \mathrm{pc}$. Measuring directly scale heights from astrometry will have to wait for Gaia (Lindegren \& de Bruijne 2005; Lindegren et al. 2009), as the Hipparcos parallaxes for late-type dwarfs are limited to $\sim 100 \mathrm{pc}$.

\section{Structure in the Galactic disk}

Eggen is often singled out as the pioneer of the study of comoving groups of stars (superclusters and moving groups) in the solar vicinity (see, e.g., Eggen 1992). Recent data sets have shown these structures with sharper contrast, and revealed new ones (see, e.g. Famaey et al. 2005; Arifyanto \& Fuchs 2006). Large surveys such as RAVE (e.g. Klement et al. 2008), and ultimately Gaia, are to provide improved statistics that will bring light on the important topic of the origin of superclusters and their connection to field stars and proper clusters.

Previous work has demonstrated that some superclusters are indeed dissolving stellar clusters (e.g., the HR 1614 moving group, which has been found to exhibit a single age and metallicity by De Silva et al. 2007). But many others that have been scrutinized appear to exhibit broad age spans (e.g. the Pleiades, Hyades, or Hercules superclusters; Famaey et al. 2008), or chemical abundance distributions (e.g. Hercules; Bensby et al. 2007), which suggests they have a dynamical origin (e.g. De Simone et al. 2004; Quillen \& Minchev 2005; Chakrabarty 2007). Note that the accretion of an external stellar system may offer, in some cases, a plausible formation scenario, with stars directly brought in with common kinematics, or simply 'linked' as a result of an accretion event (Minchev et al. 2009; Quillen et al. 2009).

\section{Ages and metallicities of disk stars}

Being able to date individual stars is extremely valuable. The recovery of the star formation history of the stars in the solar neighborhood from the inversion of observed HR diagrams or chromospheric age estimates has been attempted in many studies (RochaPinto et al. 2000; Hernández et al. 2000; Bertelli \& Nasi 2001; Aumer \& Binney 2009). Unfortunately, an examination of these and other works does not provide a coherent picture.

Isochrone dating is mostly limited to subgiants, as it is at the turn-off where the basic fundamental parameters, mainly the luminosity, change quickly, with minimal degeneracy; i.e. isochrones spread nicely. Surface gravity determinations from spectroscopy are hardly useful, since spectra are only weakly sensitive to pressure, and fundamental measurements such as trigonometric parallaxes and angular diameters are best to constrain stellar luminosities. Gaia will dramatically change this field with parallaxes accurate to $\sim 20 \mu \mathrm{m}$ at 15 magnitude.

Paying attention to details, in particular applying a rigorous statistical analysis, is important, and in some extreme cases critical. The last few years have seen a change in the methodologies for determining stellar ages, from the crude method of assigning the nearest isochrone to sophisticated statistical analyses (see, e.g., Reddy et al. 2003; Pont \& Eyer 2004; Jørgensen \& Lindegren 2005). It has been emphasized by Lachaume 

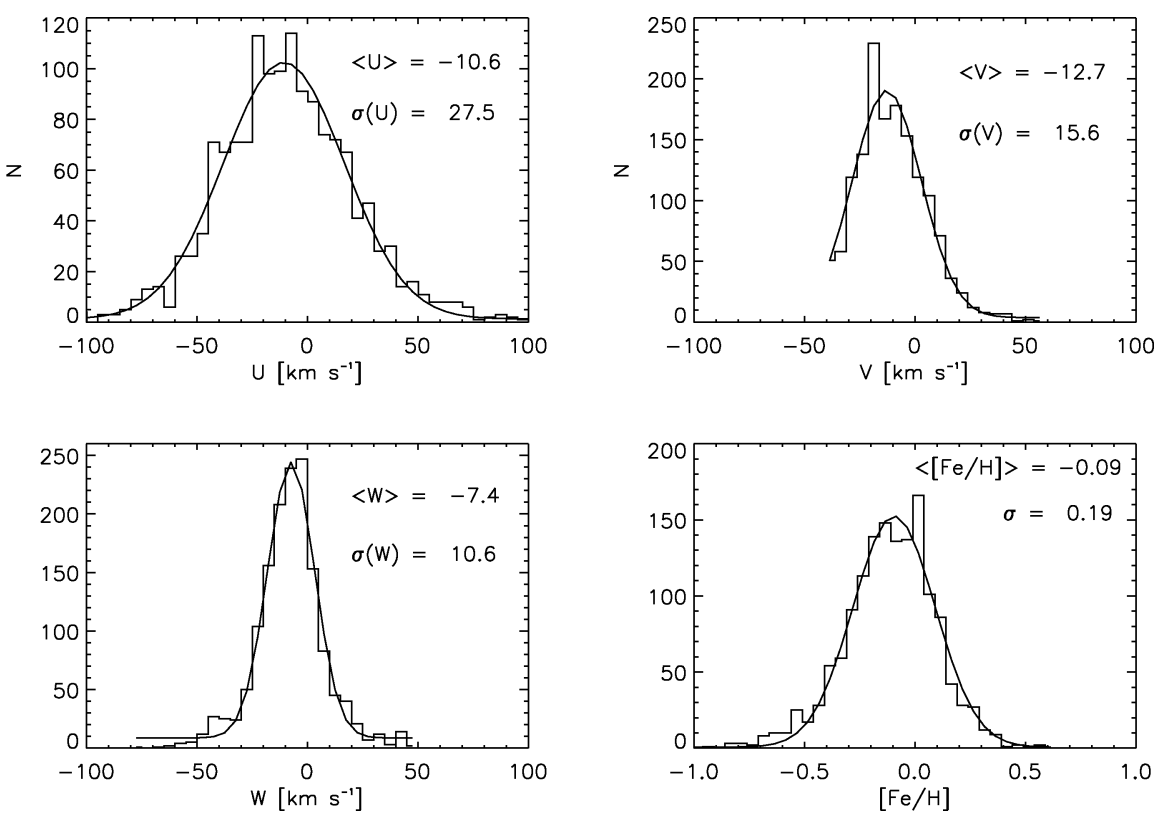

Figure 1. Velocity and metallicity histograms for 2427 stars with metallicities from the catalog of Cayrel de Strobel, Soubiran \& Ralite (2001), Hipparcos astrometry, and radial velocities from the compilations by Malaroda, Levato \& Galliani (2001) and/or Barbier-Brossat \& Figon (2000), showing velocities $V>-40$ and $-80<W<+50 \mathrm{~km} \mathrm{~s}^{-1}$. The expected contamination by thick disk and halo populations is very small, and therefore we identify the observed distributions with the thin disk. The smooth solid lines are Gaussian curves fitted to the histograms. Note the peak associated with the Hyades at $(U, V, W) \simeq(-43,-18,-2) \mathrm{km} \mathrm{s}^{-1}$. Adopted from Allende Prieto et al. (2004).

et al. (1999) that different dating techniques are complementary. For example, isochrones are most useful for turn-off stars, and hence more likely applicable to intermediate mass stars, while activity and rotation can provide ages for low mass stars that stay on the main sequence longer than a Hubble time.

Thin disk stars show a wide range of ages. Reddy et al. (2006) estimated ages between 1 and 9 Gyr, although predominantly <5 Gyr. Holmberg et al. (2009) and Haywood (2008), using larger samples, found wider ranges reaching up to 13-14 Gyr, although most concentrated again at $<4-5$ Gyr. Very old ages for thin-disk stars may be at odd with the upper limit to the age of the disk derived from the analysis of the white dwarf cooling sequence. For example, Leggett al. (1998) estimated $8 \pm 1.5$ Gyr, although a critical assessment of the literature by Fontaine, Brassard \& Bergeron (2001) led them to propose a plausible range between 8.5 to 11 Gyr.

An inspection of the literature in the last decade shows that there is now consensus regarding the metallicity distribution of the thin disk. Most authors find it is reasonably Gaussian, with a standard deviation of about 0.2 dex (see, e.g. Allende Prieto et al. 2004; Holmberg et al. 2007). More polemic is the exact mean of the distribution, which some argue could be as low as $[\mathrm{Fe} / \mathrm{H}]=-0.10$ dex, while others push for a value much close to solar (hence around 0.00; see Luck \& Heiter 2007; Haywood 2001; Taylor \& Croxall 2005; Fuhrmann 2008).

As noted some years ago, it is interesting that blindly adopting the metallicities compiled in the Cayrel de Strobel et al. catalog, and just by simply cleaning thick disk stars 
with slow Galactic rotation $\left(V<50 \mathrm{~km} \mathrm{~s}^{-1} \dagger\right)$, one recovers again a $[\mathrm{Fe} / \mathrm{H}]$ distribution centered at -0.1 dex with $\sigma \simeq 0.20 \mathrm{dex}$ - remarkably close to those found from the analysis of much more homogeneous data sets (see Fig. 1). The implication is that despite this compilation includes high-resolution determinations from many studies, the systematics across samples and analysis protocols are not large enough to widen the derived distribution.

An additional complication should be noted. Although the metallicity distribution of the thin disk may be well determined, this is likely not an ideal quantity to compare with chemical evolution models. Selection effects such as mass biases due to different lifetimes need to be considered, and so does the role of radial migration, which could be bringing significant numbers of stars formed at different galactocentric distances, and hence with different compositions even if formed at exactly the same time (see, e.g., Haywood et al. 2008).

\section{Abundance ratios}

There have been a multitude of studies performing high-resolution spectroscopy of nearby GFK stars (e.g., Edvardsson et al. 1993; Feltzing \& Gustafsson 1998; Chen et al. 2000; Nissen et al. 2000; Fulbright 2002; Reddy et al. 2003, 2006; Takeda 2007; Ecuvillon et al. 2004; Gilli et al. 2006; Bensby et al. 2003; Ramírez et al. 2007; Fuhrmann 1998, 2004, 2008). Most of these studies found a remarkable uniformity in the abundance ratios for thin disk stars at any given $[\mathrm{Fe} / \mathrm{H}]$.

Reddy et al. (2003) looked for and failed to find a cosmic scatter in the abundance ratios. Assuming $[\mathrm{Fe} / \mathrm{H}]$ is a reliable clock, the interstellar medium where these samples formed was very well mixed. Many works encounter non-solar ratios at solar $[\mathrm{Fe} / \mathrm{H}]$ for some elements. This puzzling result, which might fuel the idea of the Sun being somehow special, was later traced to systematic errors in the abundances associated with using the Sun as a reference for non-solar type stars (Allende Prieto 2008). There is no doubt that highly homogeneous samples, in particular those restricted to a narrow range in effective temperature (isothermal samples, if you will), can dramatically reduce systematic errors still present in the analyses. Meléndez et al., in these proceedings, show an extreme example of exploiting such a trick.

\section{A dichotomy between the thin and thick disks?}

The thin and thick disks stars in the solar neighborhood can be easily separated, at least statistically. Although the distributions of the velocity components and metallicities overlap somewhat, combining all the data for $U V W$ as well as $[\mathrm{Fe} / \mathrm{H}]$, makes their separation fairly straightforward. The age distributions have probably very little overlap, if any at all (see, e.g., Fig. 24 in Reddy et al. 2006). Star formation in the Milky Way has likely proceed in phases, with limited overlap: halo, thick disk, and thin disk. Yet, the connection between these three components, and in particular the thick and thin disks, is far from understood.

Looking closely at the chemical compositions, a sharp distinction between the two disks has become evident in the abundances of many elements, such as the $\alpha$-capture nuclei (O, Mg, Si, S, Ca, Ti). This is illustrated in Fig. 2, borrowed from Reddy et al.

$\dagger$ Thick disk stars lag behind the thin disk rotation by roughly that much, although this depends on the distance from the plane. 


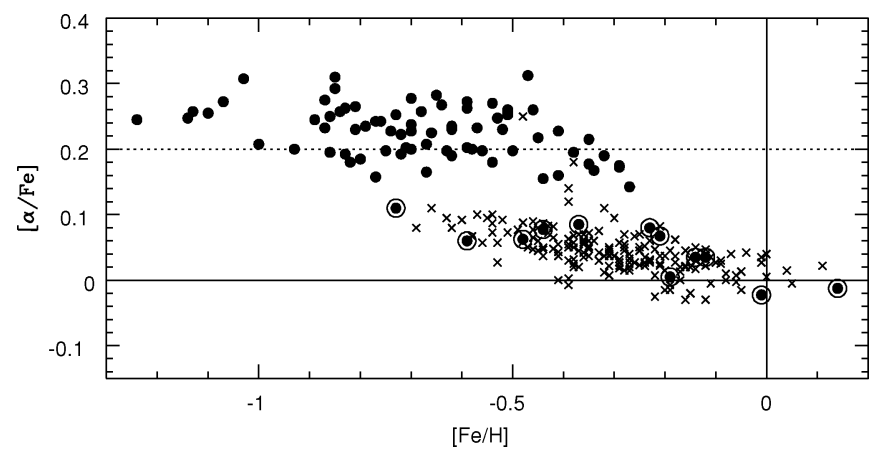

Figure 2. Average of the abundance ratios of $\mathrm{Mg}, \mathrm{Si}, \mathrm{Ca}$ and $\mathrm{Ti}$ to Fe for stars kinematically assigned to the thin (crosses) and thick disk (filled circles). The filled circles surrounded with an additional circumference are the so-called TKTA stars; they show thin-disk abundances but thick-disk kinematics. Adopted from Reddy et al. (2006).

(2006). Some argue there exists genuine transition objects, but not all studies find them in their samples.

Galactic disks can become thinner by dissipative collapse while they are still rich in gas. Such straightforward connection between the thick and thin disks does not seem viable in the light of the distinct chemical patterns that separate the two Milky Way disks. Stellar disks can also become thicker with time, due to internal (scattering and other dynamical interactions in the disk) or external (satellite accretion) mechanisms. But again, such simple path does not match the distribution of ages.

Two scenarios recently proposed in the literature appear feasible. Modelers have argued for some time that mergers could have been responsible to produce the thick disk, perhaps disrupting a previously existing disk, but allowing the thin disk to form and evolve independently afterwards. While the accretion of dry (gasless) system(s) may lead to features that clash with existing observations (think rings of stars and thick-disk characteristics that vary with galactocentric distance), the acquisition of gas rich systems and on-the-fly formation of stars seems to work well (Brook et al. 2005, 2007). The second scenario is based on new, relatively simple, models which indicate that the observed distributions of metallicity, age, and $\alpha /$ Fe ratios could emerge as a result of the natural density gradient and the associated variation of the star formation rate with galactocentric distance, when coupled to radial mixing of stellar orbits (Schönrich \& Binney 2008, 2009).

\section{The disk beyond the solar neighborhood}

The work in the solar neighborhood can now be complemented with observations of more distant stars and stellar systems. These large-range data sets will be most useful to discriminate among the proposed formation scenarios for the thick disk and its connection to thin disk and halo.

Data on individual stars from the SDSS, which now accumulates close to 0.5 million stellar spectra, can tell us about abundance and kinematics of stars over a wide range of distances, from tens to hundreds of pc using low-mass stars, to tens of kpc for bright giants. An interesting hint from SDSS is that the median of the metallicity distribution of the thick disk, about $[\mathrm{Fe} / \mathrm{H}]=-0.7$ dex, does not seem to vary between $4<R<14$ $\mathrm{kpc}$, in distinct contrast with observations for thin disk stars, where significant gradients 
are found for multiple elements using different tracers (see Fig. 13 in Allende Prieto et al. 2006).

Both Cepheids (Andrievsky et al. 2002, 2004) and giants in open clusters (Yong et al. 2006) allow tracing the abundances of many elements at large galactocentric distances. A remarkable outcome of these studies is that the well-known thin disk abundance gradient (see the review by Maciel in these proceedings) may flatten out at $R>12 \mathrm{kpc}$ (but see Sale et al. 2009 a for discrepant voice). This has been suggested to indicate a flat density profile in the inner stellar halo (Cescutti et al. 2007). Interestingly enough, these studies also show that the $\alpha / F e$ ratios increase with galactocentric distance - and so do the ratios of lanthanum (an $s$-process tracer) as well as europium (an $r$-process tracer) to iron.

The disk is by no means flat, and a better understanding of its structure is needed, in particular the flare and warp traced by stars (López-Corredoira et al. 2002; Momany et al. 2006) gas (Kalberla et al. 2007; Levine et al. 2006), and dust (Drimmel \& Spergel 2001). Infrared spectroscopic observations of vast numbers of red giants across the disk should provide much insight. APOGEE, part of SDSS-III, plans to obtain high-resolution $H$-band spectra for $10^{5}$ stars with a signal-to-noise ratio approaching 100 between 2011 and 2014 (Allende Prieto et al. 2008). Preliminary studies suggest that more than 15 chemical elements can be sampled within the $H$ band, where dust obscuration is 5 times less than in $V$.

\section{Closing remarks}

The thin disk of the Galaxy likely fits in the overall bottom-up galaxy formation scenario in a $\Lambda \mathrm{CDM}$ universe, but a detailed picture of its formation is still missing. The stellar population of the thin disk is rich in kinematic structure, but appears chemically well-mixed. The two statements in the previous sentence need not be in contradiction, as fine structure is likely missed due to limited abundance precision (currently $\sim 0.05 \mathrm{dex}$ ), and especially if most of the structure has a dynamical origin, excited by resonances and/or (modest) accretion.

The solar neighborhood needs to be placed in the context of the whole Galactic disk. Massive surveys of faint stars will do that, and they will happen over the next 5-10 years.

Among the most pressing questions in this field, we could single out: What is has been the star formation history of the solar neighborhood? (Must consider radial mixing!). Which process(es) are mainly responsible for the stellar clustering in phase space and the disk heating? What is the connection between the thin and thick disks?

The tools to address these questions are already in place: global astrometry methods, accurate spectroscopy from efficient instruments, detailed chemical analysis techniques (isothermal samples, larger samples, refined analyses), chemo-dynamical modeling, improved statistical techniques, and last but not least, data, to be provided by SDSS, RAVE, Gaia, and other supporting facilities.

\section{Acknowledgements}

I am grateful to the IAU, a Sociedade Astronômica Brasileira, and UCL graduate school for their generous support. Thanks go also to Katia Cunha for her warm hospitality, to Vivien Reuter for answering inquiries swiftly and with a smile, and to Martín López Corredoira for stimulating discussions and comments on a draft. 


\section{References}

Abazajian, K. N., et al. 2009, ApJS, 182, 543

Aguerri, J. A. L., Varela, A. M., Prieto, M., \& Muñoz-Tuñón, C. 2000, AJ, 119, 1638

Allende Prieto, C. 2008, The Metal-Rich Universe, 30

Allende Prieto, C., Beers, T. C., Wilhelm, R., Newberg, H. J., Rockosi, C. M., Yanny, B., \& Lee, Y. S. 2006, ApJ, 636, 804

Allende Prieto, C., Barklem, P. S., Lambert, D. L., \& Cunha, K. 2004, A\&AA, 420, 183

Allende Prieto, C., et al. 2008, Astronomische Nachrichten, 329, 1018

Andrievsky, S. M., Luck, R. E., Martin, P., \& Lépine, J. R. D. 2004, A\&A, 413, 159

Andrievsky, S. M., Kovtyukh, V. V., Luck, R. E., Lépine, J. R. D., Maciel, W. J., \& Beletsky, Y. V. 2002, A\&A, 392, 491

Arifyanto, M. I. \& Fuchs, B. 2006, A\& $A$, 449, 533

Aumer, M. \& Binney, J. J. 2009, MNRAS, 397, 1286

Baranne, A., Mayor, M., \& Poncet, J. L. 1979, Vistas in Astronomy, 23, 279

Barbier-Brossat, M. \& Figon, P. 2000, A\&AS, 142, 217

Bensby, T., Feltzing, S., \& Lundström, I. 2003, $A \& A$, 410, 527

Bensby, T., Oey, M. S., Feltzing, S., \& Gustafsson, B. 2007, ApJ, 655, L89

Bertelli, G. \& Nasi, E. 2001, AJ, 121, 1013

Bilir, S., Cabrera-Lavers, A., Karaali, S., Ak, S., Yaz, E., \& López-Corredoira, M. 2008, Publications of the Astronomical Society of Australia, 25, 69

Brook, C., Richard, S., Kawata, D., Martel, H., \& Gibson, B. K. 2007, ApJ, 658, 60

Brook, C. B., Gibson, B. K., Martel, H., \& Kawata, D. 2005, ApJ, 630, 298

Cabrera-Lavers, A., Bilir, S., Ak, S., Yaz, E., \& López-Corredoira, M. 2007,A\& A, 464, 565

Cayrel de Strobel, G., Soubiran, C., \& Ralite, N. 2001, A\&A, 373, 159

Cescutti, G., Matteucci, F., François, P., \& Chiappini, C. 2007, A\& $A$, 462, 943

Chakrabarty, D. 2007, A\&A, 467, 145

Chen, Y. Q., Nissen, P. E., Zhao, G., Zhang, H. W., \& Benoni, T. 2000, A\&AS, 141, 491

Dalcanton, J. J. \& Bernstein, R. A. 2002, AJ, 124, 1328

De Silva, G. M., Freeman, K. C., Bland-Hawthorn, J., Asplund, M., \& Bessell, M. S. 2007, AJ, 133,694

De Simone, R., Wu, X., \& Tremaine, S. 2004, MNRAS, 350, 627

Drimmel, R. \& Spergel, D. N. 2001, ApJ, 556, 181

Ecuvillon, A., Israelian, G., Santos, N. C., Mayor, M., Villar, V., \& Bihain, G. 2004, A\&A, 426, 619

Edvardsson, B., Andersen, J., Gustafsson, B., Lambert, D. L., Nissen, P. E., \& Tomkin, J. 1993, $A \mathscr{E} A, 275,101$

Eggen, O. J. 1992, AJ, 104, 2141

Famaey, B., Jorissen, A., Luri, X., Mayor, M., Udry, S., Dejonghe, H., \& Turon, C. 2005, Aø A, 430,165

Famaey, B., Siebert, A., \& Jorissen, A. 2008, A\& $A, 483,453$

Feltzing, S. \& Gustafsson, B. 1998, A\& AS, 129, 237

Fontaine, G., Brassard, P., \& Bergeron, P. 2001, PASP, 113, 409

Förster Schreiber, N. M., et al. 2006,ApJ, 645, 1062

Fuhrmann, K. 2004, Astronomische Nachrichten, 325, 3

Fuhrmann, K. 1998, A\& A, 338, 161

Fuhrmann, K. 2008, MNRAS, 384, 173

Fulbright, J. P. 2002, AJ, 123, 404

Gilli, G., Israelian, G., Ecuvillon, A., Santos, N. C., \& Mayor, M. 2006, A\& A, 449, 723

Gunn, J. E., et al. 1998, AJ, 116, 3040

Gunn, J. E., et al. 2006, AJ, 131, 2332

Haywood, M. 2001, MNRAS, 325, 1365

Haywood, M. 2008, MNRAS, 388, 1175

Hernandez, X., Valls-Gabaud, D., \& Gilmore, G. 2000, MNRAS, 316, 605

Holmberg, J., Nordström, B., \& Andersen, J. 2009, A $\& A$ A, 501, 941 
Holmberg, J., Nordström, B., \& Andersen, J. 2007,A\& A, 475, 519

Hopkins, P. F., Cox, T. J., Younger, J. D., \& Hernquist, L. 2009, ApJ, 691, 1168

Jurić, M., et al. 2008, ApJ, 673, 864

Jørgensen, B. R. \& Lindegren, L. 2005, A\& $A, 436,127$

Kalberla, P. M. W., Dedes, L., Kerp, J., \& Haud, U. 2007, A\&A A, 469, 511

Kauffmann, G. \& White, S. D. M. 1993, MNRAS, 261, 921

Klement, R., Fuchs, B., \& Rix, H.-W. 2008, ApJ, 685, 261

Koda, J., Milosavljević, M., \& Shapiro, P. R. 2009, ApJ, 696, 254

Koposov, S., et al. 2008, ApJ, 686, 279

Maíz-Apellániz, J. 2001, AJ, 121, 2737

Lachaume, R., Dominik, C., Lanz, T., \& Habing, H. J. 1999, AESA, 348, 897

Leggett, S. K., Ruiz, M. T., \& Bergeron, P. 1998, ApJ, 497, 294

Levine, E. S., Blitz, L., \& Heiles, C. 2006, ApJ, 643, 881

Lindegren, L., et al. 2008, IAU Symposium, 248, 217

Lindegren, L. \& de Bruijne, J. H. J. 2005, Astrometry in the Age of the Next Generation of Large Telescopes, 338, 25

López-Corredoira, M., Cabrera-Lavers, A., Garzón, F., \& Hammersley, P. L. 2002, A\& A, 394, 883

Luck, R. E. \& Heiter, U. 2007, AJ, 133, 2464

Malaroda, S., Levato, H., \& Galliani, S. 2001, VizieR Online Data Catalog, 3216, 0 (see Malaroda et al. 2000, A\&SAS, 144, 1 for more information)

Minchev, I., Quillen, A. C., Williams, M., Freeman, K. C., Nordhaus, J., Siebert, A., \& Bienaymé, O. 2009, MNRAS, 396, L56

Momany, Y., Zaggia, S., Gilmore, G., Piotto, G., Carraro, G., Bedin, L. R., \& de Angeli, F. 2006, A\&A, 451, 515

Nordström, B., et al. 2004, A\&SA, 418, 989

Onaka, P., Tonry, J. L., Isani, S., Lee, A., Uyeshiro, R., Rae, C., Robertson, L., \& Ching, G. 2008, SPIE Proc., 7014,

Pont, F. \& Eyer, L. 2004, MNRAS, 351, 487

Prieto, M., Aguerri, J. A. L., Varela, A. M., \& Muñoz-Tuñón, C. 2001, A\&A, 367, 405

Quillen, A. C. \& Minchev, I. 2005, AJ, 130, 576

Quillen, A. C., Minchev, I., Bland-Hawthorn, J., \& Haywood, M. 2009, MNRAS, 397, 1599

Ramírez, I., Allende Prieto, C., \& Lambert, D. L. 2007, A\& A, 465, 271

Reddy, B. E., Lambert, D. L., \& Allende Prieto, C. 2006, MNRAS, 367, 1329

Reddy, B. E., Tomkin, J., Lambert, D. L., \& Allende Prieto, C. 2003, MNRAS, 340, 304

Rocha-Pinto, H. J., Scalo, J., Maciel, W. J., \& Flynn, C. 2000, A\&A, 358, 869

Sale, S. E., et al. 2009, arXiv:0909.3857 (to appear in MNRAS)

Schönrich, R. \& Binney, J. 2009, MNRAS, 399, 1145

Schönrich, R. \& Binney, J. 2009, MNRAS, 396, 203

Skrutskie, M. F., et al. 2006, AJ, 131, 1163

Takeda, Y. 2007, PASJ, 59, 335

Taylor, B. J. \& Croxall, K. 2005, MNRAS, 357, 967

Toth, G. \& Ostriker, J. P. 1992, ApJ, 389, 5

West, A. A., Hawley, S. L., Bochanski, J. J., Covey, K. R., Reid, I. N., Dhital, S., Hilton, E. J., \& Masuda, M. 2008, AJ, 135, 785

West, A. A., Bochanski, J. J., Hawley, S. L., Cruz, K. L., Covey, K. R., Silvestri, N. M., Reid, I. N., \& Liebert, J. 2006, $A J, 132,2507$

West, A. A., et al. 2004, AJ, 128, 426

Yong, D., Carney, B. W., \& Teixera de Almeida, M. L. 2005, AJ, 130, 597 